\title{
Escalas de dor em crianças em idade pré-verbal e em crianças com comprometimento cognitivo e incapacidade de comunicação: uma revisão narrativa
}

\author{
Márcia Rayanne Pereira Vieira 1, Davi Soares Rodrigues 1, Liane Carvalho de Brito Souza 1, \\ Washington Aspilicueta Pinto Filho 1, 2*, Paulo Giordano Baima Colares 2, João Joaquim Freitas do \\ Amaral 1, Klenilton Lopes 1, Mariana Lima do Vale 1, Josenília Maria Alves Gomes 1, Saionara Pereira \\ da Silva 4 \\ ${ }^{1}$ Federal University of Ceara, Ceara, CE, Brazil. \\ ${ }^{2}$ Post-Graduate Program in Pharmacology, Federal University of Ceara, Ceara, CE, Brazil. \\ ${ }^{3}$ Universidade de Fortaleza (UNIFOR), Ceara, CE, Brazil. \\ ${ }^{4}$ Hospital Infantil Albert Sabin, Ceara, CE, Brazil. \\ *Corresponding author: Washington Aspilicueta Pinto Filho. Capitão Francisco Pedro Street, $n^{\circ} 1290$ - Rodolfo \\ Teófilo. Zip Code: 60430-370, Ceara, CE, Brazil. Phone: +55 (85) 3366-8220. E-mail: washifilho@hotmail.com.
}

Received on: Jul 8, 2021. Accepted on: Jul 15, 2021. Available online: Jul 19, 2021.

Abstract
O presente estudo teve por objetivo descrever escalas de avaliação da dor em
crianças com comprometimento cognitivo e incapacidade de comunicação (CCIC) e
aquelas de idade não verbal que foram e validadas no Brasil. Utilizando-se das bases
eletrônicas de dados Pubmed, MEDLINE e Google Scholar e introduzindo-se os
descritores em inglês (child or pediatric, intellectual disability, pain assessment e pain
scale), obteve-se as escalas validadas para a língua inglesa. Para os artigos, as
dissertações e as teses descrevendo escalas validadas para o Brasil sobre avaliação de
dor em crianças com CCIC ou abaixo de 4 anos de idade utilizou-se a base de dados
da BIREME, Domínio Público, Scielo, Google Scholar (artigos no Brasil) e BVS
(Biblioteca Virtual de Saúde). Foram colocados os descritores acimas citados em
português. Na literatura mundial, selecionou-se 10 escalas para avaliação de dor para
crianças com CCIC. No Brasil, apenas 3 destas estão traduzidas e validadas no nosso
meio. Para serviços pediátricos, recomenda-se utilizar as escalas que os profissionais
têm maior experiência. A escala FLACC-r (Face, Legs, Activity, Cry, Consolability-
revised) foi traduzida e validada no nosso meio e é utilizada em crianças com CCIC,
apresentando praticidade e rapidez no seu uso clínico.
Palavras-Chaves: Pediatria; Deficiência Intelectual; Medição de Dor; Comunicação
não verbal

\section{Introdução}

A dor é um sintoma comum nas enfermarias de pediatria. Estima-se que até um terço das crianças sem déficit cognitivo apresentam algum momento de dor intensa durante o internamento e a sua prevalência pode aumentar nas 
unidades cirúrgicas [1]. Esse é um problema particularmente difícil nos pacientes pediátricos com dificuldade de realizar um autorrelato confiável, condição essencial para avaliação e tratamento da condição álgica, como em recém-nascidos $(\mathrm{RN})$, crianças préverbais (abaixo de 4 anos de idade) ou portadores de síndromes genéticas e doenças neuromusculares com grave comprometimento cognitivo.

A dor pode decorrer de vários eventos, como: punções capilares, venosas, arteriais e lombares; drenagem torácica, intubação traqueal, manutenção de ventilação mecânica, aspiração traqueal, vários portes de cirurgia dentre outros. Em tais situações, observa-se baixa qualidade de atendimento, e baixo conhecimento acerca da avaliação e do tratamento da dor por parte de cuidadores e profissionais envolvidos. Muitas unidades de pediatria ainda não possuem protocolos de analgesia e sedação [2].

Até meados da década de 50, o conhecimento corrente era que as crianças, principalmente os $\mathrm{RN}$, não apresentavam desenvolvimento do Sistema Nervoso Central (SNC) suficiente para sentir dor. Entretanto, os dados atuais indicam que a dor possui um importante significado negativo nos processos de desenvolvimento neurológico e aprendizado. As vias aferentes nociceptivas se desenvolvem muito precocemente de forma que, na época do nascimento, já estão estabelecidas suas conexões com o SNC [3]. O entendimento dos mecanismos de percepção da dor ainda é um desafio em situações como crianças com déficit cognitivo.

Segundo a Organização Mundial de Saúde, a deficiência intelectual ou comprometimento de cognição é uma condição em que há um desenvolvimento incompleto ou interrompido da mente. Caracteriza-se por desabilidades de cognição, linguagem, motricidade ou social. Este prejuízo cognitivo acarreta à incapacidade ou déficit no autorrelato de suas necessidades, tornando estes indivíduos vulneráveis e dependentes. A paralisia cerebral é uma das principais causas de déficit neurológico nas crianças, as mesmas apresentam alterações do comportamento e do aprendizado, estando presente em aproximadamente dois terços destes pacientes. Estas crianças possuem maior incidência de internações e maior possibilidade de procedimentos cirúrgicos e consequentemente a presença de dor. Outros motivos de déficits neurológicos são tumores cerebrais, infartos cerebrais, traumas cranianos, doenças genéticas e suas sequelas [4].

A avaliação da dor em pediatria tem várias categorias dependendo da maturidade neurológica relacionada à idade do paciente ou a déficits neurológicos sendo estes considerados portadores de comprometimento cognitivo com incapacidade de 
comunicação (CCIC). No geral, dividese a avaliação de dor para crianças menores que um ano, crianças entre um e quatro anos (os dois últimos são considerados pré-verbais), e crianças em idade acima de cinco anos. Por definição, o autorrelato é o melhor meio de avaliar a intensidade de dor.

Em 2002, foi publicado o NonCommunicating Children's Pain ChecklistPostoperative Version (NCCPC-PV) suprindo a necessidade de avaliação de dor em crianças com déficits neurológicos após situações de dor aguda intensa. Uma vez avaliada a dor, o seu alívio, por sua vez, pode ser compreendido como um direito humano básico e, portanto, extrapola a questão clínica e vai de encontro à questão ética que envolve todos os profissionais de saúde. O problema é agravado, pois o infante não consegue reportar, somente expressar. Outro problema preponderante no controle ineficaz da dor é a falta de conhecimento acerca de sua mensuração [5].

$\mathrm{O}$ objetivo deste artigo foi descrever as escalas de avaliação de dor em crianças e adolescentes com incapacidade de comunicação por deficit cognitivo na língua inglesa. Assim como aquelas escalas para as crianças na idade pré-verbal abaixo de 4 anos e para crianças e adolescentes com CCIC utilizadas no Brasil.

\section{Results (Review)}

Foram pesquisadas as escalas de avaliação de dor em crianças com CCIC nas bases bibliográficas científicas nacionais e internacionais, Pubmed, LILACS, BIREME, Domínio Público, periódicos capes, Google Scholar, Scielo, BVS (Biblioteca Virtual de Saúde). Foram usadas de maneira total ou parcial, a depender do portal específico, os descritores em inglês e seus relativos em português brasileiro: pain assessment scale, intellectual disability, pain assessment in the cognitively impaired, pain assessment tools, pain measurement e child com base no Descritores em Ciências da Saúde (DeCS) e no Medical Subject Headings (MeSH). Os critérios de inclusão para as escalas da língua inglesa foram: instrumentos para avaliação da dor destinados a crianças e adolescentes entre 0 e 18 anos com CCIC não decorrente da idade não-verbal e publicações desde 1990.

$$
\text { Para diminuir os dados }
$$
confundidores, excluímos os artigos repetidos, os destinados a tratamentos e procedimentos, as metanálises, artigos de revisão e os destinados a idosos extremos. Especial ênfase foi dada para as escalas adaptadas ou validadas na língua portuguesa no Brasil, justificado pela limitação de escalas validadas em nossa língua, estendeu-se a pesquisa para escalas de avaliação de dor em idade não-verbal. Para esta parte da pesquisa incluiu-se artigos, monografias e capítulos de livros de texto dedicados à dor nesses pacientes. Foram individualizados 89 textos para análise. Desses estudos, foram identificadas 10 escalas internacionais que se destinam 
especificamente a este fim e 3 escalas adaptadas para nossa língua nesta metodologia. Foram acrescentadas 10 escalas utilizadas no Brasil para idade não-verbal.

As escalas internacionais identificadas foram: NCCPC-R (NonCommunicating Children Pain ChecklistRevised) [6], NCCPC-PV (Noncommunicating Children's Pain ChecklistPostoperative Version) [7], INRS (Individualized Numeric Rating Scale) [8], DESS (Douleur Enfant San Salvadour) [9], PPP (Paediatric Pain Profile) [10], FLACCr (Face, Legs, Activity, Cry, Consolabilityrevised) [11], CPB (Checklist Pain Behavior) [12], CFCS (Child Facial Coding System) [13], QPS (Questionnaire on Pain caused by Spasticity) [14], PICIC (Pain Indicator for Communicatively Impaired Children) [15].

No Brasil, apenas 3 escalas específicas para crianças com CCIC não decorrente da idade não-verbal foram traduzidas e validadas para o Brasil. Em 2015, a escala FLACC-R foi adaptada para o português brasileiro por Bussotti e colaboradores [16], a figura da escala com sua interpretação está disponível no link de sua referência: https://www.scielo.br/img/revistas/rl ae/v23n4/ /0104-1169-rlae-23-04-00651gf01.jpg [16]. A segunda escala específica para crianças com CCIC válida no Brasil é a escala PPP (Paediatric Pain Profile). Em 2011, os autores Pasin e colaboradores [17] adaptaram e validaram a escala para o Português falado no Brasil, que é uma tabela extensa de 20 itens variando de 0 a 5 pontos. Durante sua tradução e validação, a escala PPP ficou conhecida no Brasil como o Inventário de Comportamentos de Dor na Deficiência Neurológica (ICDDN) [13]. Em 2020, houve a tradução e a validação para o português brasileiro da escala NCCPCPV (Non-communicating Children's Pain Checklist-Postoperative Version). Foi a primeira escala de dor pós-operatória intencionada para crianças com CCIC [18].

Outras dez escalas de avaliação de dor estão disponíveis para nossa língua e cultura, divididas em avaliação de dor na neonatologia e em avaliação para crianças menores de idade nãoverbal. As escalas utilizadas na neonatologia podem atender à necessidade dos $\mathrm{RN}$ com déficits cognitivos. Uma parte destas escalas foram apenas traduzidas para $\mathrm{o}$ português brasileiro. Elas são comumente utilizadas na rotina de serviços de neonatologia e nos estudos científicos no nosso meio.

A citar, observamos as escalas NFCS (Sistema de Codificação da Atividade Facial Neonatal) e NIPS (Neonatal Infant Pain Scale), foram pioneiramente traduzidas pela autora brasileira Guinsburg [19]. A adaptação transcultural e validação clínica da NIPS para uso no Brasil só foi realizada por Mota e colaboradores [20]. Posteriormente, introduziu-se a escala PIPP (Premature Infant Pain Profile), que foi desenvolvida especialmente para 
avaliar a dor aguda de $\mathrm{RN}$ prematuros e de termo.

Para a avaliação da dor pósoperatória do RN, foi traduzida a escala CRIES (Crying, Requires O2 for saturation above 90\%, Increased vital signs, Expression, and Sleeplessness) [21]. Silva e colaboradores [22] realizaram uma revisão de literatura sobre a avaliação de dor em neonatologia, foram incluídas algumas escalas de dor, na referência do artigo, temos um link: https://www.scielo.br/img/revistas/r ba/v57n5/12t3.gif, demonstrando a escala CRIES junto a sua pontuação e interpretação [22].

Para as crianças com idade entre 1 e 5 anos, mesmo com CCIC, poderiam ter sua dor avaliada com auxílio de cuidador principal através de escala de faces que expressam sua dor, apesar de limitações de sua validação. Uma escala de faces de expressões dolorosas utilizando as personagens infantis da Mônica e do Cebolinha foram desenvolvidos pelo seu idealizador Maurício de Sousa, a autora Claro e colaboradores validaram este instrumento, a escala é composta por cinco expressões, as quais variam da expressão sem dor até a dor insuportável, sendo $0=$ sem dor, $1=$ dor leve, 2 = dor moderada, 3 = dor forte, 4 $=$ dor insuportável [23]. A escala CHIPPS (Children's and Infant's Postoperative Pain Scale) foi validada para crianças menores de 5 anos, sendo possível sua utilização para crianças com CCIC.
Alves e colaboradores [24] validaram esta escala no modelo de dor em crianças sem CCIC em procedimento de vacinação [24]. Outras escalas, como a escala CHEOPS (Children's Hospital of Eastern Ontario Pain Scale) que é um instrumento de 1985 válido no Canadá para crianças menores, foram traduzidas para pediatria no nosso meio [25].

As semelhanças destas duas últimas escalas são várias, como o uso do choro que pode ser de ausência, gemido ou grito, expressão facial das crianças, observa-se de relaxadas a caretas, da postura do tronco, pode ser observado uma posição neutra ou arqueado, da postura das pernas observa-se uma posição neutra, solta, chutando ou pernas tensionadas e sobre a inquietação motora da criança, podese observar nenhuma, moderada ou extrema inquietude.

Recomenda-se utilizar escalas validadas e adaptadas para o meio, a linguagem e a cultura corrente. No Brasil, algumas escalas de avaliação de dor estão devidamente estudadas e adaptadas, utilizando as idades recomendadas. As escalas citadas a seguir são utilizadas para crianças sem déficit neurológico. Entretanto muitas escalas para crianças com desabilidade de comunicação devido à idade menor poderiam ser utilizadas, estas escalas estariam adaptadas para crianças na idade pré-verbal, abaixo de 4 anos.

Os neonatos e os menores de 1 ano de vida necessitam de parâmetros 
de avaliação de dor que mescle choro, comportamento doloroso e sinais de atividade simpática. No Brasil, para crianças menores de um ano, a escala CRIES é validada para nosso meio, ela possui 5 itens de avaliação, sendo a mais utilizada para esta faixa de idade por sua rapidez e praticidade. $\mathrm{O}$ ponto de corte entre dor leve e moderada é de 5 pontos. Acima de 5 pontos, recomendase início de analgesia, pois considera-se a dor como moderada a intensa. $\mathrm{O}$ limite desta escala é de 10 pontos. São conhecidas outras escalas para avaliar a dor e o desconforto nos RN, porém poucas são validadas. Citam-se mais 3 escalas para $\mathrm{RN}$ e 1 para crianças em terapia intensiva. A escala NFCS varia pontuação de 0 a 8 pontos, sendo indicativo de dor maior que 3 pontos, a escala utiliza apenas parâmetros na expressão facial, é de rápida utilização, mas dificultosa nos pacientes em situação especial. A Escala NIPS é indicada para recém-nascidos desde idade prematura até 28 dias. Leva em consideração uma alteração fisiológica e cinco comportamentais, que transformadas em escore indicam a ausência ou presença de dor. Escores maiores do que 3 indicam presença de dor. A escala contempla a avaliação de expressão facial, choro, respiração, movimentação corporal e estado de alerta do recém-nascido. E a escala PIPP tem a característica de distinguir o RN em idades gestacionais diferentes, utiliza parâmetros comportamentais e fisiológicos, apresenta uma tabela de pontuação mais complexa, variando de 0 a 21 , os escores $\leq 6$ indicam ausência de dor ou dor mínima, escores superiores a 12 indicam a presença de dor moderada a intensa [22]. A escala de Comfort B é mais adequada para avaliar o conforto do $\mathrm{RN}$ e crianças maiores em ventilação mecânica. $\mathrm{O}$ estudo para validação no Brasil utilizou pacientes em ventilação mecânica e sob sedação, podendo ser utilizado nas crianças com CCIC. Determinou-se pontuação de corte dentro da escala Comfort B, um escore $\leq 10$ corresponderia ao excesso de sedação e $\geq 23$ à pouca sedação ou desconforto [26].

As crianças entre 1 e 4 anos terão melhor avaliação da intensidade da sensação álgica, quando a verbalização da dor for associada com o comportamento doloroso, o mesmo pode ser percebido como sinais de proteção ao local dolorido, encolhimento de tronco e pernas, caretas, enrugamento de fronte, imobilidade, inapetência ou falta de sono. As crianças com CCIC teriam parâmetros confundidores, porém a experiência prévia da criança estudada pelos profissionais e cuidadores atenuariam o fato. A escala CHIPPS já foi adaptada para nosso meio, sendo bem indicada para esta população. Em estudos internacionais, esta escala pode ser indicada para população de 0 a 4 anos, indicando um tempo de avaliação menor de 20 segundos e de fácil aplicabilidade [27]. Assim como a escala CRIES, a escala CHIPPS não necessita 
de verbalização. A transição entre dor leve e moderada é de 4 pontos. Acima de 4 pontos, considere-se a dor como moderada a intensa. Existe um limite máximo de 10 pontos. Na validação da escala CHIPPS, comparou-se a outra escala, a MBPS (Modified Behavioral Pain Scale). Houve concordância de avaliações no modelo de dor de vacinação das crianças menores [24].

A escala FLACC-r possui vários itens que descrevem o comportamento individual referentres a dor, e esta escala já está validada para nossa cultura. A escala tem 5 itens de escores de 0 a 2 pontos, sua aplicação é rápida, estima-se tempo de aplicação de 5 minutos ou menos. Ao final da tabela, observa-se orientações para seu uso, e a interpretação da soma dos escores. Esta escala tem um formato semelhante às outras escalas utilizadas para avaliar dor em crianças na idade não-verbal. É possível utilização desta pelos profissionais de saúde ou pelos cuidadores de crianças com CCIC no Brasil [16].

Já a escala ICDDN (escala PPP validada no Brasil) tem uma extensão maior, necessitaria de tempo maior para avaliação, levando a uma menor aderência nos serviços de pediatria. Os profissionais de saúde e cuidadores treinados necessitariam de tempo superior a 10 minutos para utilizar a escala. Possui 20 itens de avaliação, com escore de pontuação escalonada de 0 a 3 pontos [17].
As crianças maiores de 5 anos sem deficit de cognição são capazes de verbalizar a intensidade da dor por números, figuras, tamanhos comparativos e outras características dolorosas. O autorrelato é a melhor maneira de qualificar e quantificar a experiência dolorosa. A escala de faces de dor desenvolvida por Wong e Baker é a mais utilizada em pediatria, e necessita de verbalização dos pacientes. No entanto, as autoras desenvolveram para crianças a partir de 3 anos, que consiste em seis desenhos de faces ordenados de forma crescente em nível de intensidade da dor ou angústia, as crianças na faixa etária entre 3 e 10 anos desenharam expressões faciais em seis círculos em branco, variando de ausência de dor até a pior dor possível, na síntese do estudo, originou-se a versão final desta escala [28]. A escala verbal numérica $(E V N)$ é a forma mais comuns de avaliar a dor em adultos. A partir de EVN 4, considera-se dor moderada e EVN a partir de nota 7 é considerada como dor intensa.

Estas escalas de avaliação de dor em português falado no Brasil para crianças em idade pré-verbal e para crianças com CCIC estão sumarizadas na Tabela 1. As escalas foram qualificadas segundo presença de deficit cognitivo; faixa etária de aplicação em estudos aplicados no Brasil; modelo de estudo de validação (ambulatorial, unidade de enfermaria, terapia intensiva, pós-operatório, unidade de vacinação); uso de tradução e adaptação 
transcultural; uso de tradução livre;

autoria original e faixa etária estudada; e

autoria no Brasil.

Table 1. Escalas para avaliação de dor em pediatria em crianças e adolescentes com comprometimento cognitivo com incapacidade de comunicação (CCIC) utilizadas no Brasil.

\begin{tabular}{|c|c|c|c|c|c|c|c|}
\hline $\begin{array}{l}\text { Nome da } \\
\text { Escala }\end{array}$ & $\begin{array}{l}\text { Presença de } \\
\text { Déficit } \\
\text { Cognitivo }\end{array}$ & $\begin{array}{c}\text { Faixa } \\
\text { etária } \\
\text { em } \\
\text { estudo } \\
\text { s no } \\
\text { Brasil }\end{array}$ & $\begin{array}{c}\text { Tipo de } \\
\text { estudo e } \\
\text { local de } \\
\text { validação }\end{array}$ & $\begin{array}{l}\text { Tradução e } \\
\text { adaptação } \\
\text { transcultural }\end{array}$ & $\begin{array}{c}\text { Tradução } \\
\text { livre }\end{array}$ & $\begin{array}{c}\text { Autor } \\
\text { original e } \\
\text { faixa } \\
\text { etária } \\
\text { validada }\end{array}$ & $\begin{array}{c}\text { Autor no } \\
\text { Brasil }\end{array}$ \\
\hline FLACC-R & Sim & $\begin{array}{l}6 \text { a } 16 \\
\text { anos }\end{array}$ & $\begin{array}{l}\text { Dor pós- } \\
\text { operatória } \\
\text { (Brasil) }\end{array}$ & Sim & Não & $\begin{array}{l}{[11]} \\
-4 \text { a } 19 \\
\text { anos }\end{array}$ & [16] \\
\hline ICDDN & Sim & - & $\begin{array}{l}\text { Ambulato } \\
\text { rial, } \\
\text { enfermari } \\
\text { a e pós- } \\
\text { operatório } \\
\text {. (original- } \\
\text { Inglaterra } \\
\text { ) }\end{array}$ & Sim & Não & $\begin{array}{l}-\mathrm{PPP} \\
{[10]} \\
-1 \text { a } 18 \\
\text { anos }\end{array}$ & [17] \\
\hline NCCPC-PV & Sim & - & $\begin{array}{l}\text { Dor pós- } \\
\text { operatória } \\
\text { (original- } \\
\text { Canadá) }\end{array}$ & Sim & Não & $\begin{array}{l}{[6]} \\
-3 \text { a } 10 \\
\text { anos }\end{array}$ & [18] \\
\hline NFCS & $\begin{array}{l}\text { Pode } \\
\text { apresentar }\end{array}$ & $\begin{array}{l}\text { Neona } \\
\text { tal }\end{array}$ & $\begin{array}{l}\text { Terapia } \\
\text { intensiva } \\
\text { e } \\
\text { enfermari } \\
\text { a } \\
\text { (original- } \\
\text { Canadá) }\end{array}$ & Não & Sim & $\begin{array}{l}{[35]} \\
\text {-Neonatal }\end{array}$ & [19] \\
\hline NIPS & $\begin{array}{l}\text { Pode } \\
\text { apresentar }\end{array}$ & $\begin{array}{l}\text { Neona } \\
\text { tal }\end{array}$ & $\begin{array}{l}\text { Terapia } \\
\text { intensiva } \\
\text { (original- } \\
\text { Canadá) }\end{array}$ & Sim & Não & $\begin{array}{l}{[38]} \\
\text {-Neonatal }\end{array}$ & [20] \\
\hline PIPP & $\begin{array}{l}\text { Pode } \\
\text { apresentar }\end{array}$ & $\begin{array}{l}\text { Neona } \\
\text { tal }\end{array}$ & $\begin{array}{l}\text { Terapia } \\
\text { intensiva } \\
\text { (original- } \\
\text { Canadá) }\end{array}$ & Não & Sim & $\begin{array}{l}{[39]} \\
- \\
\text { Neonatal }\end{array}$ & [21] \\
\hline CRIES & $\begin{array}{l}\text { Pode } \\
\text { apresentar }\end{array}$ & $\begin{array}{l}\text { Até } 1 \\
\text { ano }\end{array}$ & $\begin{array}{l}\text { Terapia } \\
\text { intensiva } \\
\text { e pós- } \\
\text { operatório } \\
\text { (original- } \\
\text { EUA) }\end{array}$ & Não & Sim & $\begin{array}{l}{[37]} \\
-1 \text { a } 60 \\
\text { meses }\end{array}$ & [21] \\
\hline $\begin{array}{l}\text { Escala de faces } \\
\text { por Maurício }\end{array}$ & $\begin{array}{l}\text { Pode } \\
\text { apresentar }\end{array}$ & $\begin{array}{l}6 \text { a } 12 \\
\text { anos }\end{array}$ & $\begin{array}{l}\text { Enfermari } \\
\text { a (Brasil) }\end{array}$ & Sim & Não & $\begin{array}{l}{[28]} \\
-3 \text { a } 18\end{array}$ & [23] \\
\hline
\end{tabular}




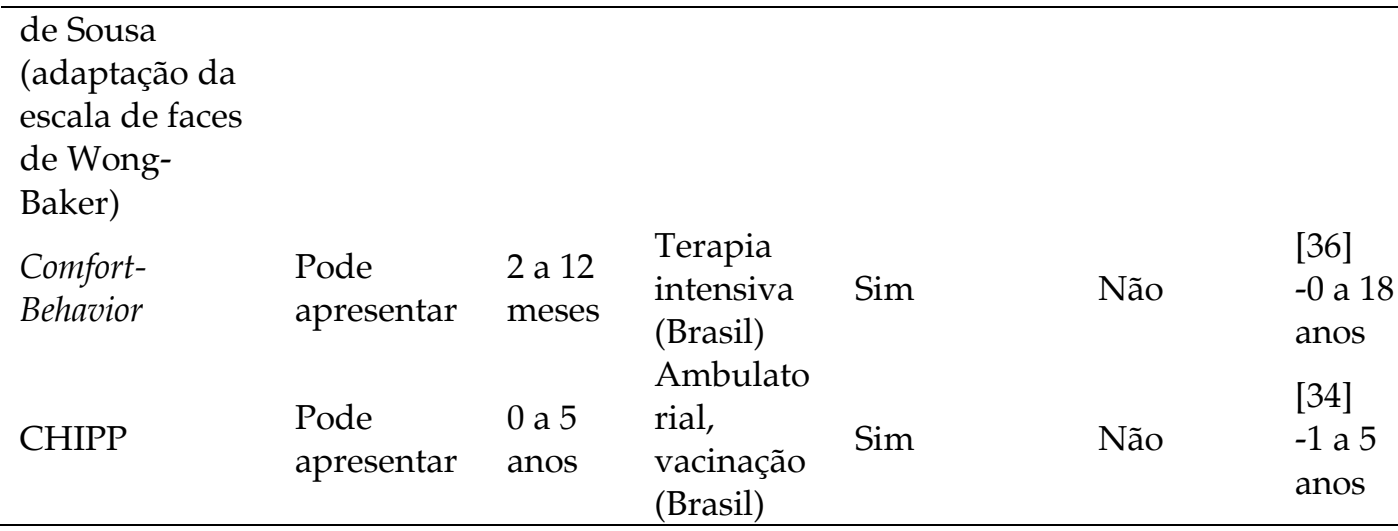

Legenda: Adaptado de Vieira e colaboradores (2021).

A avaliação acurada da intensidade da dor realizada pelo cuidador principal ou profissional de saúde responsável pela criança com CCIC é muito controversa. Num estudo avaliando esta acuidade revelou-se positivo em crianças com síndrome de Down, os pais conseguiam distinguir dor real de comportamentos semelhantes a dor [29].

Em outro estudo, observou-se uma melhor acuidade na avaliação da dor quando os pais utilizavam ferramentas específicas para avaliação em população especial, como a escala de FLACC-r. Houve menor correspondência de intensidade de dor quando utilizou-se escala numérica. E os pais superestimaram a dor de seus filhos com CCIC quando estes eram expostos a situações estressantes como cirurgia [30]. No quesito exequibilidade, num estudo comparativo entre 2 escalas, FLACC-r e PPP, a escala que demonstrou maior facilidade de preenchimento e adequação ao serviço de enfermagem foi a escala FLACC-r [31].
A responsabilidade na avaliação da dor deve ser da equipe multiprofissional, incluindo médicos, enfermeiros, cuidadores, fisioterapeuta, auxiliares, dentre outros. Todos desempenham papel importante, e a criação de equipes de dor e de cuidados especiais que podem formar protocolos e reciclar a equipe multiprofissional da unidade pediátrica.

Fora a possibilidade da avaliação comportamental, sabe-se que situações dolorosas aumentam os parâmetros fisiológicos e os hormonais. Observa-se além da alteração simpática, a alteração de salivação e concentração de cortisol em saliva. No entanto, este não é um modelo validado completamente para neonatologia e para crianças com CCIC [32]. Entretanto, já está disponível a beira do leito do paciente com auxílio de ferramentas acopladas a smartphones [33].

\section{Conclusões}

De forma geral, recomenda-se utilizar as escalas da avaliação de dor que o serviço mais se adeque, isto dependerá da experiência dos 
profissionais de saúde do local. As escalas de avaliação de dor para idade não-verbal validadas para nosso meio poderiam ser utilizadas. Porém, atualmente, temos algumas escalas para crianças e adolescentes com CCIC encontradas na literatura internacional, sendo as escalas FLACC-r, NCCPC-PV e PPP validadas para nosso meio. Elas são específicas para avaliar dor em pacientes com CCIC, sendo que a escala FLACC-r é a mais rápida e prática.

\section{References}

[1] Groenewald CB, Rabbitts JA, Schroeder DR, Harrison TE. Prevalence of moderate-severe pain in hospitalized children. Paediatr Anaesth. 2012 Jul;22(7):661-8. doi: 10.1111/j.14609592.2012.03807.x.

[2] Chermont AG, Guinsburg R, Balda $\mathrm{RC}$, Kopelman BI. O que os pediatras conhecem sobre avaliação e tratamento da dor no recém-nascido? [What do pediatricians know about pain assessment and treatment in newborn infants?]. J Pediatr (Rio J). 2003 MayJun;79(3):265-72.

[3] Mancuso T, Burns J. Ethical concerns in the management of pain in the neonate. Paediatr Anaesth. 2009 Oct;19(10):953-7. doi: 10.1111/j.14609592.2009.03144.x.

[4] Maranhão MV. Anesthesia and cerebral palsy. Rev Bras Anestesiol. 2005: 55(6):680-702.
[5] Howard RF, Lloyd-Thomas A, Thomas M, Williams DG, Saul R, Bruce E, Peters J. Nurse-controlled analgesia (NCA) following major surgery in 10,000 patients in a children's hospital. Paediatr Anaesth. 2010 Feb;20(2):126-34. doi: 10.1111/j.1460-9592.2009.03242.x.

[6] Breau LM, Finley GA, McGrath PJ, Camfield CS. Validation of the Noncommunicating Children's Pain Checklist-Postoperative Version. Anesthesiology. 2002 Mar;96(3):528-35. doi: 10.1097/00000542-200203000-00004.

[7] Breau LM, McGrath PJ, Camfield CS, Finley GA. Psychometric properties of the non-communicating children's pain checklist-revised. Pain. 2002 Sep;99(12):349-57. doi: 10.1016/s03043959(02)00179-3.

[8] Solodiuk JC, Scott-Sutherland J, Meyers M, Myette B, Shusterman C, Karian VE, Harris SK, Curley MAQ. Validation of the Individualized Numeric Rating Scale (INRS): a pain assessment tool for nonverbal children with intellectual disability. Pain. 2010 Aug;150(2):231-236. doi: 10.1016/j.pain.2010.03.016.

[9] Collignon P, Giusiano B. Validation of a pain evaluation scale for patients with severe cerebral palsy. Eur J Pain. 2001;5(4):433-42.

doi: 10.1053/eujp.2001.0265.

[10] Hunt A, Goldman A, Seers K, Crichton N, Mastroyannopoulou K, Moffat V, Oulton K, Brady M. Clinical validation of the paediatric pain profile. 
Dev Med Child Neurol. 2004 Jan;46(1):918. doi: $10.1017 /$ s0012162204000039.

[11] Malviya S, Voepel-Lewis T, Burke C, Merkel S, Tait AR. The revised FLACC observational pain tool: improved reliability and validity for pain assessment in children with cognitive impairment. Paediatr Anaesth. 2006 Mar;16(3):258-65. doi: 10.1111/j.1460-9592.2005.01773.x.

[12] Duivenvoorden HJ, Tibboel D, Koot HM, van Dijk M, Peters JW. Pain assessment in profound cognitive impaired children using the Checklist Pain Behavior; is item reduction valid? Pain. 2006 Dec 15;126(1-3):147-54. doi: 10.1016/j.pain.2006.06.025.

[13] Hadden KL, LeFort S, O'Brien M, Coyte PC, Guerriere DN. Validity of the Child Facial Coding System for the Assessment of Acute Pain in Children With Cerebral Palsy. J Child Neurol. 2016 Apr;31(5):597-602. doi: $10.1177 / 0883073815604228$.

[14] Geister TL, Quintanar-Solares M, Martin M, Aufhammer S, Asmus F. Qualitative development of the 'Questionnaire on Pain caused by Spasticity (QPS),' a pediatric patientreported outcome for spasticity-related pain in cerebral palsy. Qual Life Res. 2014 Apr;23(3):887-96. doi: $10.1007 / \mathrm{s} 11136-013-0526-2$.

[15] Stallard P, Williams L, Velleman R, Lenton S, McGrath PJ, Taylor G. The development and evaluation of the pain indicator for communicatively impaired children (PICIC). Pain. 2002 Jul;98(12):145-9.

[16] Bussotti EA, Guinsburg R, Pedreira ML. Cultural adaptation to Brazilian Portuguese of the Face, Legs, Activity, Cry, Consolability revised (FLACCr) scale of pain assessment. Rev Lat Am Enfermagem. 2015; 23(4):651-9.

[17] Pasin S, Avila F, de Cavatá T, Hunt A, Heldt E. Cross-cultural translation and adaptation to Brazilian Portuguese of the paediatric pain profile in children with severe cerebral palsy. J Pain Symptom Manage. 2013 Jan;45(1):120-8. doi: 10.1016/j.jpainsymman.2012.01.013.

[18] Colares PGB, Menezes CNB, Lima FO, Celedonio VR, Vasconcelos LMT, Gomes JMA. Translation into Brazilian Portuguese and Cross-Cultural Adaptation of the NCCPC-PV for Pain Evaluation of Patients with Intellectual Disability to Communicate. Rev Bras Ortop (Sao Paulo). 2020;55(2):170-180.

[19] Guinsburg R, Balda Rde C, Berenguel $\mathrm{RC}$, de Almeida MF, Tonelloto J, dos Santos AM, Kopelman BI. Aplicação das escalas comportamentais para a avaliação da dor em recém-nascidos [Behavioral pain scales assessment in neonates]. J Pediatr (Rio J). 1997 Nov-Dec;73(6):411-8. Portuguese. doi: 10.2223/jped.571.

[20] Motta Gde C, Schardosim JM, Cunha ML. Neonatal Infant Pain Scale: Cross-Cultural Adaptation and Validation in Brazil. J Pain Symptom 
Manage. 2015 Sep;50(3):394-401. doi: 10.1016/j.jpainsymman.2015.03.019.

[21] Guinsburg R. Avaliação e tratamento da dor no recém-nascido [Assessing and treating pain in the newborn]. J Pediatr (Rio J). 1999 MayJun;75(3):149-60. Portuguese. doi: 10.2223/jped.290.

[22] Silva YP, Gomez RS, Máximo TA, Silva ACS. Pain evaluation in neonatology. Rev Bras Anestesiol. 2007; 57(5):565-74.

[23] Claro MT. Escala de faces para avaliaçäo da dor em crianças: etapa preliminar. 1993.

[24] Alves MM, Carvalho PR, Wagner MB, Castoldi A, Becker MM, Silva CC. Cross-validation of the Children's and Infants' Postoperative Pain Scale in Brazilian Children. Pain Pract. 2008 May-Jun;8(3):171-6. doi: 10.1111/j.15332500.2008.00192.x.

[25] Oliveira RGD. Analgesia e Sedação. In: Reynaldo Gomes de Oliveira. (Org.). Black Book - Manual de Referências de Pediatria. Belo Horizonte: Black Book editora, p. 535-539, 2002.

[26] Amoretti CF, Rodrigues GO, Carvalho PRA, Trotta EA. Validação de escalas de sedação em crianças submetidas à ventilação mecânica internadas em uma unidade de terapia intensiva pediátrica terciária. Revista Brasileira de Terapia Intensiva. 2008;20: 325-330.
[27] van Dijk $M$, Peters JW, Bouwmeester NJ, Tibboel D. Are postoperative pain instruments useful for specific groups of vulnerable infants? Clin Perinatol. 2002 Sep;29(3):469-91, x. doi: 10.1016/s00955108(02)00015-5.

[28] Wong DL, Baker CM. Pain in children: comparison of assessment scales. Pediatr Nurs. 1988 JanFeb;14(1):9-17.

[29] Davies RB. Pain in children with Down syndrome: assessment and intervention by parents. Pain Manag Nurs. 2010 Dec;11(4):259-67. doi: 10.1016/j.pmn.2009.09.003.

[30] Voepel-Lewis T, Malviya S, Tait AR. Validity of parent ratings as proxy measures of pain in children with cognitive impairment. Pain Manag Nurs. 2005 Dec;6(4):168-74. doi: 10.1016/j.pmn.2005.08.004.

[31] Chen-Lim ML, Zarnowsky C, Green R, Shaffer S, Holtzer B, Ely E. Optimizing the assessment of pain in children who are cognitively impaired through the quality improvement process. J Pediatr Nurs. 2012 Dec;27(6):750-9. doi: 10.1016/j.pedn.2012.03.023.

[32] Symons FJ, ElGhazi I, Reilly BG, Barney CC, Hanson L, PanoskaltsisMortari A, Armitage IM, Wilcox GL. Can biomarkers differentiate pain and no pain subgroups of nonverbal children with cerebral palsy? A preliminary investigation based on 
noninvasive saliva sampling. Pain Med.

2015 Feb;16(2):249-56. doi: 10.1111/pme.12545.

[33] Zangheri M, Cevenini L, Anfossi L, Baggiani C, Simoni P, Di Nardo F, Roda A. A simple and compact smartphone accessory for quantitative chemiluminescence-based lateral flow immunoassay for salivary cortisol detection. Biosens Bioelectron. 2015 Feb 15;64:63-8. doi: 10.1016/j.bios.2014.08.048.

[34] Büttner W, Finke W. Analysis of behavioural and physiological parameters for the assessment of postoperative analgesic demand in newborns, infants and young children: a comprehensive report on seven consecutive studies. Paediatr Anaesth. 2000;10(3):303-18. doi: 10.1046/j.14609592.2000.00530.x.

[35] Grunau RVE, Craig KD. Pain expression in neonates: facial action and cry. Pain. 1987 Mar;28(3):395-410. doi: 10.1016/0304-3959(87)90073-X

[36] Ista E, van Dijk M, Tibboel D, de Hoog M. Assessment of sedation levels in pediatric intensive care patients can be improved by using the COMFORT "behavior" scale. Pediatr Crit Care Med. 2005 Jan;6(1):58-63. doi: 10.1097/01.PCC.0000149318.40279.1A.

[37] Krechel SW, Bildner J. CRIES: a new neonatal postoperative pain measurement score. Initial testing of validity and reliability. Paediatr
Anaesth. 1995;5(1):53-61. doi: 10.1111/j.1460-9592.1995.tb00242.x.

[38] Lawrence J, Alcock D, McGrath P, Kay J, MacMurray SB, Dulberg C. The development of a tool to assess neonatal pain. Neonatal Netw. 1993 Sep;12(6):5966.

[39] Stevens B, Johnston C, Petryshen P, Taddio A. Premature Infant Pain Profile: development and initial validation. Clin J Pain. 1996 Mar;12(1):13-22. doi: 10.1097/00002508-199603000-00004.

Conflict of interest: The author declares no conflicts of interest.

Acknowledgements: None.

Funding: None.

How to cite this article: Vieira MRP, Rodrigues DS, Souza LCB, Pinto-Filho WA, Colares PGB, Amaral JJF, Lopes K, Vale ML, Gomes JMA, Silva SP. Escalas de dor em crianças em idade pré-verbal e em crianças com comprometimento cognitivo e incapacidade de comunicação: uma revisão narrativa. Brazilian Journal of Case Reports. 2021 Jul-Sep; 01(3):14-26. 